\title{
ANÁLISE DE CONFORMIDADE EM TELHAS DE CERÂMICA VERMELHA DO NOROESTE DO RIO DE JANEIRO E DA REGIÃO LESTE DA "ZONA DA MATA MINEIRA"*
}

\author{
Niander Aguiar Cerqueira ${ }^{1}$ \\ Victor Barbosa de Souza ${ }^{2}$ \\ Afonso Rangel Garcez de Azevedo ${ }^{3}$ \\ Priscila Celebrini de Oliveira Campos ${ }^{4}$ \\ Dienifer Daiana Konzen ${ }^{5}$ \\ Victor Tomazinho Bartolazzi ${ }^{6}$ \\ Mairyanne Silva Silveira Sousa ${ }^{7}$ \\ Sergio Neves Monteiro ${ }^{8}$ \\ Fernanda Santos da $\mathrm{Luz}^{9}$
}

\section{Resumo}

Desde o período Neolítico, a argila é usada na produção de materiais para construção. Posteriormente, verificou-se que queima da argila em fornos aumentava a resistência do material, permitindo seu uso na maioria das formas abrasivas. Com a melhoria das propriedades, a cerâmica vermelha encontrou várias aplicações tais como, telhas, tijolos, louças e revestimentos. Na região estudada, a noroeste do Rio de Janeiro e a área leste da zona da mata mineira, as telhas produzidas e comercializadas exibem características correspondentes à argila local e ao método de queima. Neste trabalho foi avaliada a qualidade das telhas na região, referente às características geométricas, físicas e mecânicas, padronizadas pela norma NBR 15310:2009. De acordo com a análise dos resultados, concluiu-se que a tendência da qualidade das telhas existentes no mercado não atende aos parâmetros atuais da norma brasileira. $O$ teste geométrico apresentou o maior número de não conformidades. A planicidade da superfície e os testes de rendimento também apresentaram desvios maiores em relação ao padrão aceitável. As não conformidades encontradas nas amostras indicaram que ocorreram falhas nos controles de fabricação do produto e na aprovação do lote que liberou o material para a produção de fábrica, resultando em produtos sem padronização.

Palavras-chave: Telhas; Teste de qualidade; Inspeção; Construção civil.

\section{COMPLIANCE ANALYSIS OF ROOFING TILES IN CLAY CERAMIC IN NORTHWEST OF RIO DE JANEIRO AND THE EASTERN AREA OF "ZONA DA MATA MINEIRA" Abstract}

Since the Neolithic period, the clay is used in the production of and materials manufacturing of building. After that, it was verified that the fired clays in furnaces showed higher resistance allowing their use in most abrasive ways. This improving of properties enabled the several use of ceramic such as tiles, bricks and coatings. In the region studied, northwest of Rio de Janeiro and the eastern area of "zona da mata mineira", the tiles produced and commercialized exhibit characteristics corresponding to local clay and the firing method. In this work it was evaluated the quality of these ceramic tiles, relative to geometrical, physical and mechanical characteristics, standardized by NBR 15310:2009. According to analysis of the results, it was conclude that the trend of the quality of existing ceramic tiles in the market is not meeting the current parameters of the Brazilian standard. The geometric test had the highest number of non-conformities. The surface flatness and the yield tests also showed higher deviations in relation to the acceptable standard. The non-conformities found in the samples indicate that there were controls failures in the product manufacturing and batch approval that releases the material to the factory output resulting in products without standardization.

Keywords: Ceramic roofing tiles; Quality testing; Inspection; Buildings. 
1 Engenheiro Civil, D.Sc., Departamento de Engenharia Civil, Universidade Estadual do Norte Fluminense Darcy Ribeiro, UENF, Brasil.

2 Engenheiro Mecânico, M.Sc., Doutorando em Engenharia Mecânica, Universidade Federal Fluminense, UFF, Brasil.

3 Engenheiro Civil, M.Sc., Doutorando em Engenharia Civil, Universidade Estadual do Norte Fluminense Darcy Ribeiro, Campos dos Goytacazes, UENF, RJ, Brasil. afonso.garcez91@gmail.com

4 Engenheira Civil, Mestranda em Engenharia de Transportes, Instituto Militar de Engenharia, Rio de Janeiro, RJ, Brasil.

5 Graduanda em Engenharia Civil, Faculdade Redentor, FACREDENTOR, Itaperuna, RJ, Brasil.

6 Tecnólogo em informática, M.Sc., Professor do Departamento de Engenharia de Produção, Faculdade Redentor, FACREDENTOR, Itaperuna, RJ, Brasil.

7 Engenheira Civil, Mestranda em Engenharia Civil, Universidade Estadual do Norte Fluminense Darcy Ribeiro, UENF, Brasil.

8 Engenheiro Metalurgista, Ph.D, Professor Titular, Seção de Engenharia Mecânica e de Materiais, Instituto Militar de Engenharia, Rio de Janeiro, RJ, Brasil.

9 Engenheira de Materiais, M.C, Doutoranda em Ciência dos Materiais, Seção de Engenharia Mecânica e de Materiais, Instituto Militar de Engenharia, Rio de Janeiro, RJ, Brasil. fsl.santos@gmail.com 


\section{INTRODUÇÃO}

No noroeste do Rio de Janeiro e na área leste da "zona da mata mineira", as telhas cerâmicas produzidas e comercializadas exibem características correspondentes da argila local e o método de queima.

De acordo com os dados do IBGE [1], "os municípios que compõem a área de estudo têm suas atividades orientadas essencialmente para a agricultura e a atividade de mineração é considerada secundária. Entre os bens minerais explorados, destacam-se os materiais de construção civil, principalmente a rocha decorativa (que alimenta o mercado local) e os mármores para a indústria do cimento (que atende os estados do Rio de Janeiro, Minas Gerais e Espírito Santo)". $\mathrm{Na}$ área de Itaperuna, há apenas uma mina na região de Bom Jesus de Itabapoana, ao longo do rio Muriaé [2]. Provavelmente é um tipo de terraço-depósito aluvial do Rio Itabapoana.

No registro do DNPM [3], existe uma mina em atividade em sedimentos quaternários do rio Muriaé em Italva. Nesta região existe um conjunto de empresas que exploram o mármore para fabricação de cimento. Estas argilas podem ser derivadas da alteração do desgaste dos mármores.

A indústria de cerâmica local tem produtos de baixa produtividade, alto custo e não padronizados. Assim, a atividade mineira e a produção de cerâmica vermelha se retiraram cada vez mais no estado do Rio de Janeiro, pressionando o mercado para importações de outras regiões.

Este trabalho teve como objetivo estudar os resultados da caracterização de vários tipos de telhas comercializadas regionalmente de acordo com a norma técnica atual NBR 15310: 2009 [4].

\section{MATERIAIS E MÉTODOS}

Para este estudo, foi desenvolvido um levantamento prévio dos principais tipos de telhas vendidos na região. Com esses dados, foram coletadas seis amostras do mesmo lote de quatro modelos distintos para determinar as características desejadas e compará-las com as especificações da norma ABNT- NBR 15310:2009 [4].

Os quatro modelos mais encontrados foram:

1: Telha Paulista: caracteriza-se por ter a cobertura um pouco menor do que a largura do canal. Fornece um movimento de plástico diferenciado para os telhados;

2: Telha plana: é uma variação de cobertura e canal, que tem caminho reto;

3: Telha Romana: apresenta baixo custo e é um dos modelos mais populares. Uma forma característica que se encaixa longitudinalmente e transversalmente. Este tipo de telha foi padronizada recentemente em 1996;

4: Telha Portuguesa: ainda não padronizada pela norma teve seu uso consolidado na construção civil devido ao seu design atraente, fácil escoamento de água da chuva, bom alinhamento e alta economia na colocação [5].

As telhas foram compradas em lojas regionais específicas e analisadas no laboratório da Faculdade Redentor, Campus Itaperuna/RJ.

Os testes foram realizados seguindo as especificações da norma NBR 15310:2009 [4].

Os aspectos analisados foram:

- Acústico e visual; 
- Geométrico: comprimento de fabricação $(L)$, largura $(W)$, posição do pino $\left(L_{p}\right)$, altura do pino $\left(\mathrm{H}_{\mathrm{p}}\right)$, rendimento $\left(\mathrm{Y}_{\mathrm{a}}\right)$ e planicidade da superfície.

- Funcional e tecnológica: impermeabilidade e absorção de água.

\subsection{Análise visual e acústica}

A norma NBR 13818 [6] define que a cerâmica deve apresentar alguns aspectos perceptíveis a olho nu a uma distância de um metro, tais como: bordas finas e suaves; brilho; uniformidade de cor; queima adequada e uniforme; textura lisa; e ausência de rebarbas, rachaduras, quebras e esfoliação corporal.

No teste acústico, as amostras foram suspensas, presas em sua extremidade mais larga e com um objeto metálico seu lado oposto foi golpeado. $\mathrm{O}$ som da batida deve ter um caráter metálico, caso contrário não está em conformidade.

\subsection{Análise geométrica}

De acordo com a NBR 15310 [4], as amostras foram colocadas em superfície plana e suportadas em suas extremidades. Com a ajuda de calibres cada lado da telha foi medido. O comprimento médio e a largura de uma telha oblonga consistem na média das medidas de dois lados opostos.

A planicidade foi determinada pelo maior desvio em função da medida obtida dos três pontos da superfície da telha.

Para o teste de rendimento, foram organizadas cinco amostras telhas, um conjunto no centro e os outros ao redor. A telha central foi substituída quatro vezes. Com os valores médios de largura e comprimentos foi calculada a área útil $\left(A_{u}\right)$, a partir da qual se obteve o rendimento médio $\left(\mathrm{Y}_{\mathrm{a}}\right)$, dado pela Equação (1).

$$
\mathbf{Y}_{\mathbf{a}}=\frac{\mathbf{1}}{\mathrm{A}_{\mathbf{u}}}
$$

\subsection{Aspectos funcionais e tecnológicos}

Para avaliar a impermeabilidade, as amostras foram colocadas em posição horizontal e elevadas pelas extremidades. Foi colocado na sua superfície um quadro selado que ocupa $65 \%$ da superfície da telha juntamente com uma coluna constante de água no seu interior. As amostras foram mantidas por um dia neste estado. Finalmente, foi verificada se houve presença de gotas na face inferior, as quais indicam não conformidade, e o aparecimento de manchas de umidade que são toleradas.

No teste de absorção de água, as telhas cerâmicas foram colocadas em uma temperatura do forno de $100^{\circ} \mathrm{C}$, durante 24 horas, e removidas para a medição do seu peso seco. Em seguida, essas telhas foram submersas em um tanque de água durante 24 horas. A porcentagem de absorção de água foi obtida através do aumento da massa das amostras após imersão, conforme a Equação (2):

$$
\mathbf{W} \%=\frac{\mathbf{M}_{2}-\mathbf{M}_{1}}{\mathbf{M}_{1}} \times \mathbf{1 0 0}
$$

Sendo $M_{2}$ o peso da amostra após 24 horas de imersão em água limpa e $M_{1}$ o peso da amostra seca. 


\section{RESULTADOS E DISCUSSÃO}

\subsection{Análise visual e acústica}

A norma NBR 15310 [4] determina que as telhas podem apresentar ocorrências de quebras, esfoliações, lascados e rebarbas desde que não prejudiquem seu desempenho.

A Tabela 1 mostra os resultados para análise visual e acústica.

Tabela 1. Qualidade da superfície da telha.

\begin{tabular}{|c|c|c|c|}
\hline Amostra & Conformidade & Não conformidade & \% de telhas no lote \\
\hline Plana & 40 & 0 & 100,0 \\
Paulista & 38 & 2 & 95,0 \\
Romana & 37 & 3 & 92,5 \\
Portuguesa & 38 & 2 & 95,0 \\
\hline \% de telhas aceitas no lote & \multicolumn{3}{|c}{} \\
\hline
\end{tabular}

Apenas as telhas Romanas foram reprovadas pelo teste visual. No teste de acústico, todas as amostras de todos os tipos testados apresentaram um som de caráter metálico, sendo assim, aprovados.

\subsection{Análise geométrica}

Conforme a norma, a tolerância dimensional permitida é de $\pm 2,0 \%$ para as dimensões de fabricação. Para as telhas prensadas, o pino deve ter uma altura mínima $\left(\mathrm{H}_{\mathrm{p}}\right)$ de $7 \mathrm{~mm}$ e para telhas extrudadas a altura mínima é de $3 \mathrm{~mm}$. A tolerância aceitável para o valor do rendimento $\left(\mathrm{Y}_{\mathrm{a}}\right)$ é $\pm 1 \%$ [4].

$\mathrm{Na}$ Tabela 2 estão apresentados os resultados para os aspectos geométricos das telhas extrudadas.

Tabela 2. Aspectos geométricos das telhas extrudadas.

\begin{tabular}{|c|c|c|c|c|c|c|c|c|c|}
\hline \multirow{2}{*}{\multicolumn{2}{|c|}{ Telhas }} & \multicolumn{4}{|c|}{ Plana } & \multicolumn{4}{|c|}{ Paulista } \\
\hline & & $\mathbf{L}$ & W & $L_{p}$ & $\mathbf{H}_{\mathrm{p}}$ & $\mathbf{L}$ & W & $\mathbf{L}_{p}$ & $\mathrm{H}_{\mathrm{p}}$ \\
\hline \multirow{6}{*}{ Amostra } & 1 & 465,0 & 130,0 & 434,0 & 5,0 & 450,0 & 160,0 & 416,0 & 6,0 \\
\hline & 2 & 463,0 & 124,0 & 434,0 & 5,0 & 450,0 & 161,0 & 415,0 & 7,0 \\
\hline & 3 & 464,0 & 128,0 & 433,0 & 5,0 & 445,0 & 162,0 & 413,0 & 6,0 \\
\hline & 4 & 464,0 & 128,0 & 434,0 & 5,0 & 450,0 & 160,0 & 418,0 & 6,0 \\
\hline & 5 & 465,0 & 130,0 & 435,0 & 5,0 & 446,0 & 152,0 & 412,0 & 6,0 \\
\hline & 6 & 466,0 & 128,0 & 435,0 & 5,0 & 450,0 & 161,0 & 418,0 & 7,0 \\
\hline \multicolumn{2}{|c|}{ Média das amostras $(\mathrm{mm})$} & 464,5 & 128,0 & 434,2 & 5,0 & 448,5 & 161,0 & 415,3 & 6,3 \\
\hline \multicolumn{2}{|c|}{ Tamanho médio (mm) } & 460,0 & 130,0 & 434,0 & 8,0 & 450,0 & 160,0 & 424,0 & 8,0 \\
\hline \multicolumn{2}{|c|}{$\begin{array}{l}\text { Desvio aceitável para o } \\
\text { tamanho médio }\end{array}$} & \multicolumn{3}{|c|}{$2 \%$} & $\geq 3 \mathrm{~mm}$ & \multicolumn{3}{|c|}{$2 \%$} & $\geq 3 \mathrm{~mm}$ \\
\hline
\end{tabular}


De acordo com as especificações da norma, apenas uma amostra da telha Plana apresentou não conformidade, sendo esse tipo aprovado. Porém, as telhas do tipo Paulista apresentam quatro amostras que excederam o desvio aceitável pela norma. A média do tamanho das amostras também se mostraram não conformes, assim, os aspectos geométricos deste tipo foram reprovados.

A Tabela 3 mostra os resultados para os aspectos geométricos das telhas cerâmicas prensadas.

Tabela 3. Aspectos geométricos das telhas prensadas a seco.

\begin{tabular}{|c|c|c|c|c|c|c|c|c|c|}
\hline \multirow{2}{*}{\multicolumn{2}{|c|}{ Telhas }} & \multicolumn{4}{|c|}{ Portuguesa } & \multicolumn{4}{|c|}{ Romana } \\
\hline & & $\mathbf{L}$ & w & $L_{p}$ & $H_{p}$ & $\mathbf{L}$ & w & $L_{p}$ & $\mathbf{H}_{\mathrm{p}}$ \\
\hline \multirow{6}{*}{ Amostras } & 1 & 400,0 & 230,0 & 370,0 & 5,0 & 403,0 & 233,0 & 380,0 & 5,0 \\
\hline & 2 & 405,0 & 235,0 & 374,0 & 6,0 & 402,0 & 232,0 & 380,0 & 5,0 \\
\hline & 3 & 404,0 & 232,0 & 374,0 & 5,0 & 403,0 & 232,0 & 380,0 & 5,0 \\
\hline & 4 & 400,0 & 233,0 & 370,0 & 6,0 & 401,0 & 232,0 & 381,0 & 5,0 \\
\hline & 5 & 400,0 & 230,0 & 370,0 & 6,0 & 402,0 & 231,0 & 381,0 & 5,0 \\
\hline & 6 & 400,0 & 230,0 & 370,0 & 6,0 & 400,0 & 232,0 & 376,0 & 5,0 \\
\hline \multicolumn{2}{|c|}{$\begin{array}{l}\text { Média das amostras } \\
(\mathrm{mm})\end{array}$} & 401,5 & 231,7 & 371,3 & 5,7 & 401,8 & 232,0 & 379,7 & 5,0 \\
\hline \multicolumn{2}{|c|}{ Tamanho médio(mm) } & 400,0 & 232,0 & 377,0 & 8,0 & 400,0 & 230,0 & 365,0 & 8,0 \\
\hline \multicolumn{2}{|c|}{$\begin{array}{c}\text { Desvio aceitável para o } \\
\text { tamanho médio }\end{array}$} & \multicolumn{2}{|c|}{$2 \%$} & \multicolumn{2}{|c|}{$\geq 7 \mathrm{~mm}$} & \multicolumn{2}{|c|}{$2 \%$} & \multicolumn{2}{|c|}{$\geq 7 \mathrm{~mm}$} \\
\hline
\end{tabular}

As amostras prensadas a seco apresentaram medidas de comprimento e largura conforme ao padrão existente. No entanto, a altura do pino foi menor, sendo essas amostras reprovadas.

\subsection{Planicidade}

Como especificado em norma, os valores encontrados na medida da planicidade não devem exceder $5 \mathrm{~mm}$, independentemente do tipo de telha [4].

$\mathrm{Na}$ Tabela 4 são mostrados os resultados da verificação de planicidade da superfície dos diferentes tipos de telhas.

Tabela 4. Planicidade da superfície.

\begin{tabular}{|c|c|c|}
\hline $\begin{array}{c}\text { Amostras } \\
\text { (6 unidades) }\end{array}$ & $\begin{array}{c}\text { Média } \\
(\mathbf{m m})\end{array}$ & $\begin{array}{c}\text { Telhas não conformes no lote } \\
\text { (unidades) }\end{array}$ \\
\hline Plana & 4,83 & 3 \\
Paulista & 6,17 & 3 \\
Romana & 3,00 & - \\
Portuguesa & 7,00 & 5 \\
\hline \% de telhas aceitas no lote & \multicolumn{2}{|c|}{ mínimo de 95 } \\
\hline
\end{tabular}


Neste requisito (Tab. 4), somente as telhas Romanas foram aprovadas neste teste.

\subsection{Rendimento}

O rendimento é expresso em unidade de telha por $\mathrm{m}^{2}\left(\mathrm{~T} / \mathrm{m}^{2}\right)$, no qual é permitido um coeficiente de variação de 1\% [5]. Para este teste, não foram avaliadas as telhas extrudadas.

A Tabela 5 apresenta os resultados de rendimento das telhas prensadas a seco.

Tabela 5. Rendimento das telhas prensadas a seco.

\begin{tabular}{|c|c|c|c|c|c|c|c|c|}
\hline \multirow{2}{*}{$\begin{array}{c}\text { Amostra } \\
\text { central }\end{array}$} & $\begin{array}{c}\text { Comp. } \\
\text { médio } \\
\text { útil } \\
(\mathbf{m m})\end{array}$ & $\begin{array}{c}\text { Largura } \\
\text { média } \\
\text { útil } \\
(\mathbf{m m})\end{array}$ & $\begin{array}{c}\text { Área } \\
\text { média } \\
\text { útil } \\
\left(\mathbf{m m}^{2}\right)\end{array}$ & $\mathbf{Y}_{\mathrm{a}} \mathbf{( T / \mathbf { m } ^ { 2 } )}$ & $\begin{array}{c}\text { Comp. } \\
\text { médio } \\
\text { útil } \\
(\mathbf{m m})\end{array}$ & $\begin{array}{c}\text { Largura } \\
\text { média } \\
\text { útil } \\
(\mathbf{m m})\end{array}$ & $\begin{array}{c}\text { Área } \\
\text { média } \\
\text { útil } \\
\left(\mathbf{m m}^{2}\right)\end{array}$ & $\mathbf{Y}_{\mathrm{a}}\left(\mathbf{T} / \mathbf{m}^{2}\right)$ \\
\hline 1 & 350,00 & 150,50 & 52675,0 & 18,5 & 335,50 & 166,50 & 55861,0 & 17,9 \\
\hline 2 & 345,00 & 152,50 & 52613,0 & 18,8 & 340,00 & 158,50 & 53890,0 & 18,6 \\
\hline 3 & 344,00 & 152,00 & 52288,0 & 19,1 & 337,50 & 156,50 & 52819,0 & 18,8 \\
\hline 4 & 347,50 & 153,00 & 53168,0 & 18,8 & 339,50 & 155,50 & 52793,0 & 19,2 \\
\hline 5 & 346,63 & 152,00 & 52686,0 & 19,4 & 338,13 & 159,30 & 53840,0 & 18,6 \\
\hline \multicolumn{3}{|c|}{ Rendimento médio } & 18,92 & & - & & 18,62 \\
\hline \multicolumn{6}{|c|}{ Desvio permitido do rendimento } \\
médio & & $1 \%$ & \\
\hline
\end{tabular}

Com base nos resultados obtidos, todas as amostras apresentaram rendimento superior ao especificado.

\subsection{Aspectos funcionais e tecnológicos}

No teste de impermeabilidade todos os tipos de telhas foram aceitos, assim como, para o teste de absorção de água, no qual foi considerado um limite de absorção de água de $20 \%$, visto que a área de estudo se enquadra no clima temperado/tropical.

A Tabela 6 mostra os resultados de absorção de água.

Tabela 6. Absorção de água.

\begin{tabular}{|l|c|c|c|c|}
\hline \multicolumn{2}{|c|}{ Amostras (6 unidades) } & $\begin{array}{c}\text { Massa seca - } \mathbf{M}_{\mathbf{1}} \\
\mathbf{( g )}\end{array}$ & $\begin{array}{c}\text { Massa úmida - } \\
\mathbf{M}_{\mathbf{2}} \mathbf{( g )}\end{array}$ & Absorção W\% \\
\hline \multirow{3}{*}{ Média } & Plana & $2.029,87$ & $2.477,27$ & $18,06 \%$ \\
& Paulista & $1.875,03$ & $2.295,97$ & $18,33 \%$ \\
& Romana & $2.407,10$ & $2.954,53$ & $18,53 \%$ \\
& Portuguesa & $2.540,70$ & $3.050,87$ & $16,72 \%$ \\
\hline
\end{tabular}

Observando os resultados dessa tabela conclui-se que todas as amostras foram aprovadas neste requisito.

A Figura 1 apresenta um gráfico das não conformidades detectadas em cada um dos testes para todos os tipos de amostras ensaiadas. 


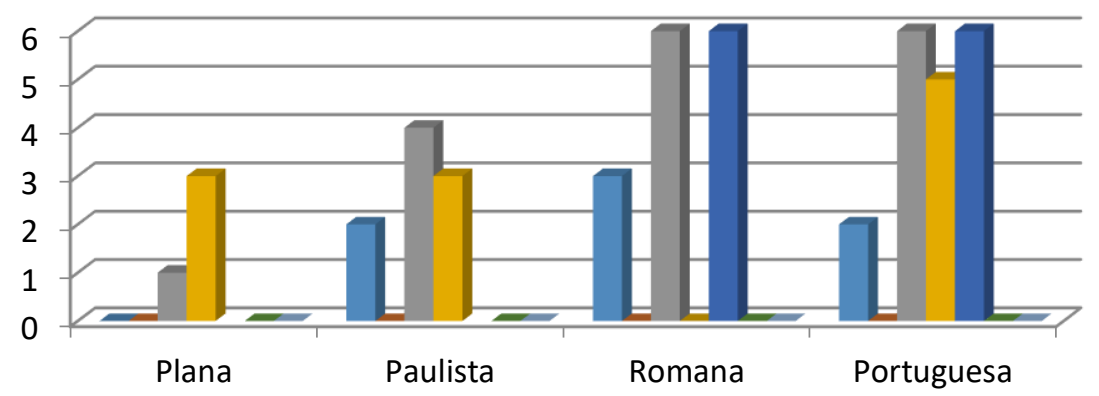

Qualidade superficial

- Teste acústico

Aspestos geométricos

- Planicidade

Rendimento

- Impermiabilidade

Figura 1. Número de não conformidades detectadas em cada um dos testes para os diferentes tipos de telhas.

Com base nesse gráfico, conclui-se que o teste geométrico teve o maior número de não conformidades, onde apenas as telhas planas foram aprovadas neste requisito, mostrando a tendência de não conformidade das telhas existentes no mercado. Referente aos testes de planicidade e rendimento, os resultados apresentaram os maiores desvios em relação ao padrão aceitável, indicando falha nos controles de fabricação e aprovação dos produtos liberados para a comercialização.

\section{CONCLUSÃo}

De acordo com a análise dos resultados, as não conformidades encontradas nas amostras indicaram a ocorrência de falha nos controles de fabricação do produto e aprovação do lote liberado, tendo como resultado produtos sem padronização disponibilizados para os consumidores. Portanto, conclui-se que há uma tendência da qualidade das telhas existentes no mercado não atenderem aos parâmetros atuais estabelecidos pela norma brasileira.

\section{Agradecimentos}

Os autores deste trabalho agradecem ao CNPq, à FAPERJ e à CAPES pelo financiamento da pesquisa.

\section{REFERÊNCIAS}

[1] IBGE. Instituto Brasileiro de Geografia e Estatística. Produto interno bruto 2004-2008. Rio de Janeiro: IBGE. 2010.

[2] Duarte B.P. et al. Geologia e recursos minerais da folha Itaperuna SF.24-V-C-I, estado do Rio de Janeiro. Belo Horizonte:CPRM, 2012.

[3] Departamento Nacional de Produção Mineral. Sumário Mineral 2008 - Ministério de Minas e Energia, Departamento Nacional de Produção Mineral. Brasília : DNPM/DIPLAM, 2012.

[4] Associação Brasileira de Normas Técnicas. ABNT NBR 15310: Componentes cerâmicos, telhas - Terminologia, requisitos e métodos de ensaio. Rio de Janeiro: ABNT, 2009.

[5] Associação Brasileira de Normas Técnicas. ABNT NBR 13818: Placas cerâmicas para revestimento - Especificação e métodos de ensaios. Rio de Janeiro: ABNT, 1997. 